\title{
Aberrant Methylation of the 1p36 Tumor Suppressor Gene RIZ1 in Renal Cell Carcinoma
}

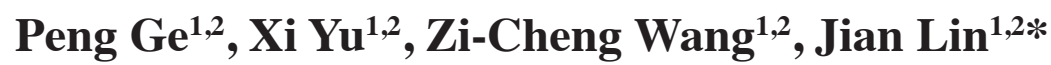

\begin{abstract}
Background: Retinoblastoma protein-interacting zinc finger gene $1(R I Z 1)$ functions as a tumor suppressor. Hypermethylation-mediated RIZ1 silencing has been reported in several cancers, but not in renal cell carcinoma (RCC) yet. Materials and Methods: We examined the RIZ1 expression and methylation in a panel of RCC cell lines and 50 primary tumors using semiquantitative/quantitative polymerase chain reaction (PCR), methylation specific PCR, and bisulfite sequencing genomic. We also explored the relationship between methylation status of RIZ1 and clinicopathological features in RCC patients. Results: RIZ1 expression was down-regulated or lost in OS-RC-2, 769-P, Caki-1, 786-O and A498 RCC cell lines. Restored expression of RIZ1 was detected after addition of 5-aza-2'-deoxycytidine with/without trichostatin A, suggesting that DNA methylation directly mediates its silencing. The RIZ1 expression was significantly reduced in RCCs compared to adjacent non-malignant renal samples $(P<0.001)$. Aberrant methylation was detected in 15 of 50 (30\%) RCCs and in 2 of 28 (7\%) adjacent nonmalignant renal samples $(\boldsymbol{P}=\mathbf{0 . 0 2})$. No statistically significant correlation between methylated and unmethylated cases with regard to age, gender, pathological stage and grade was observed. Conclusions: RIZ1 expression is down-regulated in human RCC, and this down-regulation is associated with methylation. RIZ1 methylation may play a role in renal carcinogenesis.
\end{abstract}

Keywords: DNA methylation - RIZ1 - tumor suppressor gene - kidney neoplasms - epigenetics

Asian Pac J Cancer Prev, 16 (9), 4071-4075

\section{Introduction}

Renal cell carcinoma (RCC) accounts for more than $90 \%$ of all kidney malignancies, with the highest incidence occurring in Western countries. RCC treads to have a long asymptomatic latency period in early stage. Most RCCs are found during radiological investigation coincidentally (Hauser et al., 2013). Roughly 33\% patients present with metastatic disease at the first diagnostic procedure and a significant proportion of patients experience low treatment efficacy (Zhang et al., 2010). Hence, insights into the molecular mechanisms of tumorigenesis underlying RCC are necessary for identifying reliable biomarker for its early detection and prognosis.

Like other tumors, RCC involves complex, multistep and heterogeneous malignant carcinogenicity duo to accumulated genetic and epigenetic alterations (Knudson, 2001). Dissimilarly, mutation does not represent a frequent event in RCC (Zhang et al., 2014). Current knowledge regarding molecular mechanisms indicates that DNA methylation serves as a major mechanism in mediating tumor suppressor genes (TSGs) silencing and its silencing can be a fundamental event in nephrocarcinogenesis (Esteller, 2007; Hoffman et al., 2011). It is well established that epigenetic changes can be detected earlier than genetic alterations in tumorigenesis (Gnyszka et al., 2013). Additionally, aberrant methylation represents an early event in cancer formation (Miyamoto et al., 2005). Together, these results suggest a promising value of DNA methylation for early diagnosis and prognosis (de Martino et al., 2012; Raja et al., 2012; Hauser et al., 2013; Haroun et al., 2014). A wealth of genes have been described as methylation targets in RCC, such as DAPK, SPINT2, APC, RASSF1A, VHL, $\gamma$-catenin, P16 and CDH1 (Zhang et al., 2007; Zhang et al., 2010; Hoffman et al., 2011; Zhang et al., 2014). However, most of these TSGs represent a relative low rate of DNA methylation. Therefore, further study stressing on novel, specific TSGs silenced by promoter methylation is required.

Retinoblastoma protein-interacting zinc finger gene (RIZ or PRDM2), located on $1 \mathrm{p} 36$, is a member of the nuclear protein/histone methyltransferase (MTase) superfamily (Tokumaru et al., 2003). This superfamily of gene is functionally involved in gene expression, in development, and in cancer (Huang et al., 1998; Tokumaru et al., 2003). The $R I Z$ gene contains a novel protein MTase domain, termed the PR domain (a conserved positive regulatory domain; also called the SET domain), and normally encodes two types of protein products with different length through alternative promoters, RIZ1 and 
RIZ2 (Lal et al., 2006; Dong et al., 2012). RIZ1 contains a PR domain and is thought to have tumor-suppressive propertles (Du et al., 2001). However, RIZ2 lacks this domain. The expression and activity of RIZ1, but not of $R I Z 2$, are commonly decreased or lost in a considerable proportion of cancers (He et al., 1998; Du et al., 2001; Sasaki et al., 2002; Hasegawa et al., 2007). Previous studies have shown that promoter methylation serves as a common mechanism in mediating the RIZl gene silencing in leukemia, prostate cancer, breast cancer, and gastric cancer, and this silencing can significantly contribute to human carcinogenesis (He et al., 1998; Du et al., 2001; Steele-Perkins et al., 2001; Sasaki et al., 2002; Hasegawa et al., 2007). However, the role of RIZ1 gene in human $\mathrm{RCC}$ remains unclear.

In this study, we aimed to examine the expression and promoter methylation status of RIZ1 in RCC, and to explore the relationship between methylation status of RIZ1 and clinicopathological features in RCC patients.

\section{Materials and Methods}

\section{Cell lines and drug treatment}

Five RCC cell lines including OS-RC-2, 769-P, Caki1,786-O, and A498, and the immortalized human normal embryonic kidney cell line HEK293 were studies. They were routinely maintained in Dulbecco's modified Eagle's medium or RPMI 1640 medium supplemented with $10 \%$ fetal bovine serum, and $1 \%$ penicillin-streptomycin and incubated in a humidified atmosphere with $5 \%$ $\mathrm{CO}_{2}$ at $37^{\circ} \mathrm{C}$. Cell lines with silenced RIZl expression (OS-RC-2, 786-0, and 769-P) were treated with $10 \mu \mathrm{M}$ DNA demethylating agent 5-aza-2'-deoxycytidine (Aza) (Sigma, ST Louis, USA) with or without $100 \mathrm{nM}$ histone deacetylase inhibitor Trichostatin A (TSA) (Sigma, ST Louis, USA), as described previously (Zhang et al., 2014). Cells were then harvested for further analysis.

\section{Patients and tissue samples}

A total of 50 primary RCCs and 28 matched adjacent non-malignant renal samples were obtained with informed consent from the department of urology, Peking University First Hospital, Beijing, China. No cases had adjuvant therapy prior to surgery. All RCCs were validated pathologically. Histopathological grade and stage were assigned according to the Fuhrman nuclear grade and the 2002 American Joint Committee on Cancer (AJCC) recommendations, respectively (Greene et al., 2002; Eble et al., 2004). Table 1 shows the clinicopathological features of RCC patients.

RNA extraction, semiquantitative RT-PCR and quantitative real-time $P C R$

Total RNA was isolated from cell lines and tissues using TRI REAGENT (Invitrogen, Carlsbad, USA). The first strand was synthesized from $1 \mu \mathrm{g}$ total RNA using AMV Reverse Transcriptase (Promega, Madison, WI). The expression of RIZl in cell lines and tissues was detected by reverse transcriptase PCR (RT-PCR) with GoTaq (Promega, Madison, WI) and/or by quantitative real-time PCR (qRT-PCR) (7500 Fast Real-Time PCR
System, Applied Biosytems) with TransStart ${ }^{\circledR}$ Green qPCR SuperMix (TransGen, Beijing, PRC) according to the manufacturer's recommendations. GAPDH was applied as an internal control (Dong et al., 2012). All qRTPCR amplifications were performed in triplicate. The $\Delta \mathrm{Ct}$ for mRNA expression of RIZl was calculahted relative to the $\mathrm{Ct}$ of $G A P D H$. The primers and cycling parameters were published previously (Dong et al., 2012).

\section{DNA extraction, bisulfite treatment and methylation analysis}

Extraction of genomic DNA was carried out using TIANamp Genomic DNA Kit (TIANGEN, Beijing, PRC) and bisulfite modification of genomic DNA was performed using EpiTect Fast DNA Bisulfite Kit (Qiagen, Hilden, GER) according to the manufacturer's protocols, respectively. Methylation specific PCR (MSP) and bisulfite genomic sequencing (BGS)was applied to detect the promoter methylation status of RIZI using the primers described previously (Du et al., 2001). The PCR primers generated for MSP covered eight common methylated CpGs (Du et al., 2001). The MSP reaction products were electrophoresed on a $20 \mathrm{~g} / \mathrm{L}$ agarose gel and subsequently semi-quantified by UV spectrophotometer (Gene, Shanghai, PRC). Amplified BGS products were cloned into a T5 vector according to the manufacturer's protocols (TransGen, Beijing, PRC). 5 to 8 colonies were randomly isolated and sequenced (Lal et al., 2006; Zhang et al., 2010; Zhang et al., 2014).

\section{Statistical analysis}

Associations of categorical variables were assessed using chi-square test. Differences in continuous variables were analyzed using the Wilcoxon signed-rank test or Student's t test. Statistical analyses were all performed using SAS version 9.2 (SAS Institute, Cary, NC, USA). All $P$-values were based on two-sided tests and a $P$-value of less than 0.05 was considered statistically significant.

\section{Results}

RIZ1 is frequently silenced in RCC cell lines by promoter methylation

First of all, we examined the expression of RIZ1 in 5 RCC cell lines and the immortalized human normal embryonic kidney cell line HEK293 using RT-PCR. All cell lines were silenced or down-regulated compared with HEK293 (Figure 1A), indicating that RIZ1 is a candidate TSG in RCC. In addition, the RIZ1 promoter consists of a typical $\mathrm{CpG}$ island, suggesting that RIZ1 inactivating might be mediated by epigenetic alternative mechanism. Thus we investigated the RIZ1 promoter methylation using MSP and BGS. MSP analysis revealed that the promoter of RIZ1 was methylated in all RCC cell lines (Figure 1A). In contrast, obvious RIZl methylation was not found in HEK293. In order to confirm the MSP results, we examined the RIZ1 promoter methylation status using BGS. High density of methylated $\mathrm{CpG}$ sites were found in 769-P cell line (Figure 2A). Taken together, these results suggest RIZ1 gene expression level is closely correlated with its promoter methylation status inversely. 

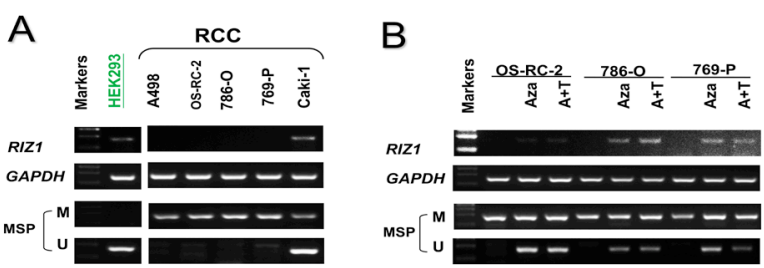

C

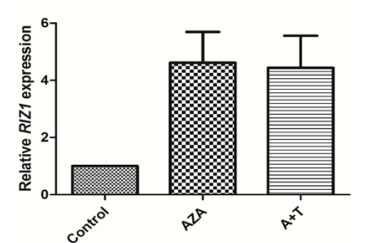

D

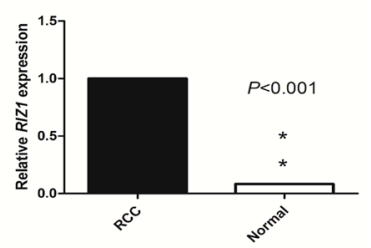

Figure 1.RIZ1 Expression and Methylation-mediated RIZ1 Expression Silencing. (A) Detection of RIZI expression in a panel of RCC cell lines by RT-PCR. M, methylated; U, unmethylated. (B) Pharmacological demethylation using 5-aza-2'-deoxycytidine(Aza) with/without Trichostatin A(TSA) restored RIZl expression in RCC cell lines. A+T, Aza and TSA. (C) Detection of RIZ1 expression of OS-RC by qRT-PCR after addition of Aza with or without TSA. Control, 786-O without Aza or TSA; A+T, Aza and TSA. (D) RIZ1 expression in RCC samples relative to matched adjacent non-malignant renal samples. The median relative RIZ1 expression of 'normal' was 0.08 (interquartile range: $0.03-0.50$ ). Normal, matched adjacent non-malignant renal samples


Figure 2. BGS Analysis in Cell Lines and MSP Results of Primary Tumors. (A) Bisulfite genomic sequencing(BGS) analysis of the RIZ1 promoter in HEK293 and RCC 769-P cell lines. (B) BGS analysis of 786-O treated with or without Aza. (C) Methylation specific PCR (MSP) results of RIZ1 methylation in 24 primary tumors. M, methylated; $\mathrm{U}$, unmethylated

\section{Pharmacological demethylation reactivates RIZ1 expression}

To examine whether methylation directly mediates RIZ1 inactivating, we treated silenced and methylated RCC cell lines, OS-RC-2, 786-O, and 769-P, using Aza with or without TSA. Restored expression of RIZ1 was detected by RT-PCR after treatment with Aza in these cell lines, along with an increase of unmethylated alleles by MSP (Figure 1B). Similar results were observed in cell lines treated with Aza and TSA $(\mathrm{A}+\mathrm{T})$. Of note, the expression of RIZl of OS-RC-2 was still weak after drug treatment. We further examined the expression level of RIZl of OS-RC-2 using qRT-PCR. An obvious increase of

Table 1. Clinicopathological Features in Patients with RCC and RIZ1 Methylation Status

\begin{tabular}{|c|c|c|c|}
\hline & $\begin{array}{c}\text { No. Methylated } \\
(\%)\end{array}$ & $\begin{array}{c}\text { No. Unmethylated } \\
(\%)\end{array}$ & $P$-value \\
\hline Overall & $15(30.0)$ & $35(70.0)$ & \\
\hline Age & $58.1 \pm 13.2$ & $55.9 \pm 13.9$ & $0.6^{\mathrm{a}}$ \\
\hline Gender & & & $1.00^{\mathrm{b}}$ \\
\hline Male & $11(28.9)$ & $27(71.1)$ & \\
\hline Female & $4(33.3)$ & $8(66.7)$ & \\
\hline \multicolumn{3}{|c|}{ Pathological stage $^{1}$} & $0.45^{\mathrm{c}}$ \\
\hline $\mathrm{T} 1$ & $7(21.2)$ & $26(78.8)$ & \\
\hline $\mathrm{T} 2$ & $3(42.9)$ & $4(57.1)$ & \\
\hline $\mathrm{T} 3$ & $3(42.9)$ & $4(57.1)$ & \\
\hline T4 & $1(50.0)$ & $1(50.0)$ & \\
\hline \multicolumn{3}{|c|}{ Nuclear grade $^{1}$} & $0.51^{\mathrm{c}}$ \\
\hline G1 & $2(25.0)$ & $6(75.0)$ & \\
\hline G2 & $8(25.0)$ & $24(75.0)$ & \\
\hline G3 & $4(44.4)$ & $5(55.6)$ & \\
\hline
\end{tabular}

*149 available; aStudent's t test; ' Mantel-Haenszel Chi-Square; 'CochranMantel-Haenszel Statistics

RIZ1 expression level was found between the drug treated groups (Aza with or without TSA) and the untreated group ( $\sim 4$ folds, Figure 1C). High-resolution BGS analysis revealed a decreased percentage of RIZI methylation from $76.6 \%$ to $44.0 \%$ in Aza treated 786-O cells (Figure $2 \mathrm{~B})$. These results indicate that aberrant $R I Z 1$ promoter methylation mediates its silencing in RCC.

\section{RIZ1 mRNA expression in paired samples}

We compared the RIZ1 mRNA contents between RCC and corresponding adjacent non-malignant tissues for 20 patients by qRT-PCR. Overall, the RIZ1 mRNA expression level of tumor samples compared to the correspondings was dramatically decreased (median fold difference $=0.08$, $P<0.001$, Figure 1D). In addition, to get a preliminary impression on whether the RIZ1 expression varies with tumor progression (Sun et al., 2011), we compared the mRNA expression levels of RIZ1 in early stages (T1 and T2) and advanced stages (T3 and T4). There was no statistically significant variation $(P=0.19)$.

\section{Frequent methylation of RIZ1 in primary tumors}

We further investigated the RIZI promoter methylation status in 50 primary kidney cancers and 28 matched adjacent non-malignant renal samples. Table 1 and Figure $2 \mathrm{C}$ show the clinicopathological features and the MSP results, respectively. The ratio of male to female of these patients was $3.2(38 / 12)$, and the mean age was 56.5 (range: $28-81$, standard deviation: 13.6). We found RIZ1 methylation in 15 of 50 (30\%) RCCs. In the nonmalignant samples, only $7 \%$ ( 2 of 28 ) were detected with weak methylation. Statistical analysis of this variation revealed a significant difference $(P=0.02)$.

We also explored the relationship between methylation state of RIZ1 and clinicopathological features in RCC patients. There was no statistically significant correlation between methylated patients and unmethylated patients with regard to age, gender, pathological stage and grade (Table 1). 


\section{Discussion}

In this current study, we investigated the RIZ1 promoter methylation status in RCC cell lines and tumor tissues. Our results indicate that the RIZ1 expression is decreased or lost in renal cancer cell lines as well as tissues and such decreased or lost RIZ1 expression is largely regulated by promoter methylation. These results suggest that RIZ1 may be a potential tumor suppressor gene in $\mathrm{RCC}$, as in other malignancies.

$R I Z$ was initially isolated by Buyse (1995) in a functional screening for Rb-binding proteins. RIZl functions as a TSG because of harboring the PR domain (Sun et al., 2011). It catalyzes histone H3K9 methylation leading to repression of transcription (Xie et al., 1997). It is well established that histone methylation plays an important role in suppressing cancer, indicating that aberrant inactivation of $R I Z 1$ can be a critical mechanism for cancer formation and progression (Du et al., 2001). The expression of $R I Z 1$ is down-regulated in many human malignancies (He et al., 1998; Du et al., 2001; Sasaki et al., 2002; Hasegawa et al., 2007; Pastural et al., 2007). Although a wealth of genes are silenced in human cancers, $R I Z 1$ is one the few that have been proved to be causally linked with carcinogenesis (Du et al., 2001; Steele-Perkins et al., 2001). Du (2001) firstly showed that promoter methylation resulted in loss of RIZ1 mRNA expression. Subsequently, other studies confirmed this notion that promoter methylation serves as a common mechanism in mediating the RIZl gene silencing (Lal et al., 2006; Cheng et al., 2010; Dong et al., 2012). In prostate cancer, Hasegawa (2007) reported that $42.6 \%$ of cancer cases had RIZ1 methylation and methylation correlated with high Gleason score, $53.3 \%$ in high score vs. $23.5 \%$ in low score, respectively. In bladder cancer, $9.8 \%$ of cancer tissues were observed to have methylation (Garcia-Baquero et al., 2013).

To the best of our knowledge, this is the first study to investigate the methylation status of RIZI in RCC and its relationship with clinicopathological information. In this study, we found that the RIZI expression was reduced or lost in RCC cell lines as well as tissues. All the 5 RCC cell lines OS-RC-2, 769-P, Caki-1, 786-O, and A498 showed reduced $R I Z 1$ expression and methylation. The promoter methylation status was closely correlated with decreased RIZl gene expression. Restored expression of RIZ1 was detected after addition of Aza with/without TSA in the silenced and methylated renal cancer cell lines OS-RC-2, 786-O, and 769-P. However, both genetic and epigenetic mechanisms can result in inactivating TSGs. In addition, $R I Z 1$ is located at $1 \mathrm{p} 36$, a region commonly deleted in a variety of human cancers (Pastural et al., 2007). To assess the potential impact of genetic alterations on RIZ1 expression, we examined the COSMIC database for RIZ1 genetic mutations (PRDM2, search time: October 13, 2014) (Zhang et al., 2014). Overall, there were 776 kidney cancer samples tested for RIZ1 mutations and only $3(0.39 \%)$ showed point mutation. Typically, our results revealed that $30 \%$ kidney cancer samples had RIZ1 methylation. Studies have shown that TSGs tend to be preferentially silenced by a preferred mechanism (Du et al., 2001). This indicates that although RIZ1 localizes to a region which commonly undergoes deletion, RIZ1 might be preferentially inactivated by DNA methylation in RCC, "a preferred mechanism". Similarly, promoter hypermethylation has been shown to be the most common mechanism resulting in reduced $R I Z I$ expression in other types of cancers (Khaenam et al., 2010). However, we cannot exclude other potential mechanisms that can lead to $R I Z 1$ silencing. One such potential mechanism is that $R I Z 1$ inactivating could result from a defect of a certain transcription factor that normally activates the $R I Z 1$ promoter (Du et al., 2001). Given the prevalence of RIZI promoter methylation, however, this potential is not likely to be commonly involved.

Aberrant methylation represents an early molecular event in tumourigenesis, indicating a promising potential for early diagnosis (Miyamoto et al., 2005). In our series, we found RIZ1 methylation in $30 \%(15 / 50)$ kidney cancer samples. In matched adjacent non-malignant renal samples, 2 cases were reported to have methylation, suggesting that RIZ1 methylation occurs early in carcinogenesis. This finding corroborates a previous study in which methylation was found in precancerous lesions (Tokumaru et al., 2003). The sensitivity and specificity of RIZ1 methylation for diagnosing RCC were $30 \%$ and $93 \%$, respectively. Although the sensitivity was low, the specificity was excellent. Given the lack of a reliable biomarker for kidney cancer diagnosis, further study stressing on examining the diagnostic value of RIZ1 methylation in body fluids is required. Hauser ( 2013) analyzed the diagnostic value of DNA promoter methylation of six genes (APC, GSTE1, P14, PTGS2, $R A R-B$, and $R A S S F 1 A$ ) and the sensitivity of a single gene ranged from $14.3 \%$ to $54.3 \%$. Another study conducted by de Martino (2012) reported similar results. In this study, 4 genes were tested (VHL, RASSF1A, PTGS2, and P16) and the sensitivity of a single gene ranged from $38.2 \%$ to $50.3 \%$. Of note, duo to the biologic heterogeneity of kidney cancer, a single biomarker may not be sufficient to assist in clinical decision making. A combination of methylation biomarkers of several genes will likely improve overall predictive accuracy (de Martino et al., 2012; Hauser et al., 2013).

Methylation may also reflect the severity of disease (Zhang et al., 2010; Zhang et al., 2014), and thus it could be a prognostic marker. Zhang (2010) reported that DLEC1 methylation was significantly associated with pathological stage and grade. Additionally, similar results were published on RASSFIA and IRF8 (Ellinger et al., 2011; Zhang et al., 2014). In this study, we did not observe statistically significant correlation between methylated patients and unmethylated patients with regard to pathological stage and grade. However, the statistical power may be weakened by our small sample size. More investigations with a large number of samples are warranted to validate these conclusions.

The vital role of epigenetic alterations in cancer initiation and development has been realized by human beings. Fortunately, unlike genetic mutations, epigenetic alterations are potentially reversible. Therefore, such alterations provide novel opportunities for cancer therapy. 
Currently, several DNA methyltransferase inhibitors have been used in clinical practice for patients with hematological malignancies and some solid tumors, including azacytidine and decitabine (Gnyszka et al., 2013). Promisingly, further studies on DNA methylation of genes may provide a new therapeutic target for RCCs unresponsive to conventional therapies.

In conclusion, our study represents the first documentation to investigate the RIZl promoter methylation status in RCC cell lines and tumor tissues. Our results indicate that the $R I Z 1$ expression is decreased or lost in renal cancer cell lines as well as tissues and such decreased or lost RIZ1 expression is largely regulated by promoter methylation. Epigenetic silencing of RIZI may have diagnostic value of early detection for RCC. Further study is needed to elucidate the mechanisms of RIZ1 involved in renal carcinogenesis.

\section{References}

Buyse IM, Shao G, Huang S (1995). The retinoblastoma protein binds to RIZ, a zinc-finger protein that shares an epitope with the adenovirus E1A protein. Proc Natl Acad Sci U S A, 92, 4467-71.

Cheng HY, Gao Y, Lou G (2010). DNA methylation of the RIZ1 tumor suppressor gene plays an important role in the tumorigenesis of cervical cancer. Eur J Med Res, 15, 20-4.

de Martino M, Klatte T, Haitel A, Marberger M (2012). Serum cell-free DNA in renal cell carcinoma: a diagnostic and prognostic marker. Cancer, 118, 82-90.

Dong SW, Zhang P, Liu YM, et al (2012). Study on RIZ1 gene promoter methylation status in human esophageal squamous cell carcinoma. World J Gastroenterol, 18, 576-82.

Du Y, Carling T, Fang W, et al (2001). Hypermethylation in human cancers of the RIZ1 tumor suppressor gene, a member of a histone/protein methyltransferase superfamily. Cancer Res, 61, 8094-9.

Eble JN, Epstein JI, Sesterhenn IA (2004). Pathology and genetics of tumors of the urinary system and male genital organs. IARC Press, Lyon.

Ellinger J, Holl D, Nuhn P, et al (2011). DNA hypermethylation in papillary renal cell carcinoma. BJU Int, 107, 664-9.

Esteller M (2007). Cancer epigenomics: DNA methylomes and histone-modification maps. Nat Rev Genet, 8, 286-98.

Garcia-Baquero R, Puerta P, Beltran M, et al (2013). Methylation of a novel panel of tumor suppressor genes in urine moves forward noninvasive diagnosis and prognosis of bladder cancer: a 2-center prospective study. J Urol, 190, 723-30.

Gnyszka A, Jastrzebski Z, Flis S (2013). DNA methyltransferase inhibitors and their emerging role in epigenetic therapy of cancer. Anticancer Res, 33, 2989-96.

Greene FL, Page DL, Fleming ID, et al (2002). AJCC cancer staging manual, 6th edn. Springer publisher, New York.

Haroun RA,Zakhary NI, Mohamed MR, et al (2014). Assessment of the prognostic value of methylation status and expression levels of FHIT, GSTP1 and p16 in non-small cell lung cancer in Egyptian patients. Asian Pac J Cancer Prev, 15, 4281-7.

Hasegawa Y, Matsubara A, Teishima J, et al (2007). DNA methylation of the RIZl gene is associated with nuclear accumulation of $\mathrm{p} 53$ in prostate cancer. Cancer Sci, 98, 32-6.

Hauser S, Zahalka T, Fechner G, et al (2013). Serum DNA hypermethylation in patients with kidney cancer: results of a prospective study. Anticancer Res, 33, 4651-6.

He L, Yu JX, Liu L, et al (1998). RIZ1, but not the alternative $R I Z 2$ product of the same gene, is underexpressed in breast cancer, and forced RIZ1 expression causes G2-M cell cycle arrest and/or apoptosis. Cancer Res, 58, 4238-44.

Hoffman AM, Cairns P (2011). Epigenetics of kidney cancer and bladder cancer. Epigenomics, 3, 19-34.

Huang S, Shao G, Liu L (1998). The PR domain of the Rbbinding zinc finger protein $R I Z 1$ is a protein binding interface and is related to the SET domain functioning in chromatinmediated gene expression. J Biol Chem, 273, 15933-9.

Khaenam P, Jearanaikoon P, Pairojkul C, et al (2010). Genetic and epigenetic alterations of RIZ1 and the correlation to clinicopathological parameters in liver fluke-related cholangiocarcinoma. Exp Ther Med, 1, 385-390.

Knudson AG (2001). Two genetic hits (more or less) to cancer. Nat Rev Cancer, 1, 157-62.

Lal G, Padmanabha L, Smith BJ, et al (2006). RIZ1 is epigenetically inactivated by promoter hypermethylation in thyroid carcinoma. Cancer, 107, 2752-9.

Miyamoto K, Ushijima T (2005). Diagnostic and therapeutic applications of epigenetics. Jpn J Clin Oncol, 35, 293-301.

Pastural E, Takahashi N, Dong WF, et al (2007). RIZ1 repression is associated with insulin-like growth factor-1 signaling activation in chronic myeloid leukemia cell lines. Oncogene, 26, 1586-94.

Raja UM, Gopal G, Rajkumar T (2012). Intragenic DNA methylation concomitant with repression of ATP4B and ATP4A gene expression in gastric cancer is a potential serum biomarker. Asian Pac J Cancer Prev, 13, 5563-8.

Sasaki O, Meguro K, Tohmiya Y, et al (2002). Altered expression of retinoblastoma protein-interacting zinc finger gene, RIZ, in human leukaemia. Br J Haematol, 119, 940-8.

Steele-Perkins G, Fang W, Yang XH, et al (2001). Tumor formation and inactivation of RIZ1, an Rb-binding member of a nuclear protein-methyltransferase superfamily. Genes Dev, 15, 2250-62.

Sun W, Qiao L, Liu Q, et al (2011). Anticancer activity of the PR domain of tumor suppressor RIZ1. Int J Med Sci, 8, 161-7.

Tokumaru Y, Nomoto S, Jeronimo C, et al (2003). Biallelic inactivation of the RIZ1 gene in human gastric cancer. Oncogene, 22, 6954-8.

Xie M, Shao G, Buyse IM, Huang S (1997). Transcriptional repression mediated by the PR domain zinc finger gene RIZ. J Biol Chem, 272, 26360-6.

Zhang Q, Ying J, Li J, et al (2010). Aberrant promoter methylation of DLEC1, a critical 3p22 tumor suppressor for renal cell carcinoma, is associated with more advanced tumor stage. J Urol, 184, 731-7.

Zhang Q, Ying J, Zhang K, et al (2007). Aberrant methylation of the 8 p22 tumor suppressor gene DLC1 in renal cell carcinoma. Cancer Lett, 249, 220-6.

Zhang Q, Zhang L, Li L, et al (2014). Interferon regulatory factor 8 functions as a tumor suppressor in renal cell carcinoma and its promoter methylation is associated with patient poor prognosis. Cancer Lett, 354, 227-34. 